\title{
Local Flow Field and Slip Length of Superhydrophobic Surfaces
}

\author{
David Schäffel, Kaloian Koynov, Doris Vollmer, Hans-Jürgen Butt, and Clarissa Schönecker* \\ Max Planck Institute for Polymer Research, 55128 Mainz, Germany \\ (Received 15 September 2015; revised manuscript received 8 December 2015; published 30 March 2016)

\begin{abstract}
While the global slippage of water past superhydrophobic surfaces has attracted wide interest, the local distribution of slip still remains unclear. Using fluorescence correlation spectroscopy, we performed detailed measurements of the local flow field and slip length for water in the Cassie state on a microstructured superhydrophobic surface. We revealed that the local slip length is finite, nonconstant, anisotropic, and sensitive to the presence of surfactants. In combination with numerical calculations of the flow, we can explain all these properties by the local hydrodynamics.
\end{abstract}

DOI: 10.1103/PhysRevLett.116.134501

The question of whether and how a Newtonian fluid is able to slip over a solid surface has arisen with the development of theoretical models for fluid motion at the beginning of the 19th century. Because of its high fundamental and technological importance, it has been continuously studied and controversially discussed ever since. Still, it is not yet fully answered. Following Navier [1], slippage is typically characterized by the slip length $b$, relating velocity $u$, and stress at the surface via the boundary condition $u=b(\partial u / \partial n)$ with $n$ being the normal to the surface. While the intrinsic slip on smooth, hydrophobic surfaces has been found to be below $50 \mathrm{~nm}$ [2-9], a fundamental understanding of the presumably much larger and therefore technologically much more relevant apparent slip on rough surfaces is still missing. This type of slip is due to a fluid being in the Cassie state; i.e., for typical superhydrophobic surfaces, air is entrapped underneath the water in surface indentations. In this case, slip may be considered on two length scales: first, the effective slip that represents the inhomogeneous surface by an averaging parameter and thereby characterizes the flow far from the surface. Most experiments are limited to this global viewpoint, e.g., by considering a net drag reduction [10-18]. Second, slip may be considered at the length scale of the surface inhomogeneities. To date, slippery surfaces are typically modeled by applying a Navier slip condition locally at the position of the fluid-fluid interfaces that is supposed to represent the mechanisms in the lubricating layer. This local slip condition is typically strongly idealizing, treating the air-water interfaces either as perfectly slipping without any resistance [19-25], with a constant local slip length $[26,27]$ or with a generic, isotropic distribution [28-30], although these properties have not

Published by the American Physical Society under the terms of the Creative Commons Attribution 3.0 License. Further distribution of this work must maintain attribution to the author(s) and the published article's title, journal citation, and DOI. yet been measured nor do they contain information about the specific surface design, e.g., the geometry of the roughness. Bolognesi, Cottin-Bizonne, and Pirat found the local slip length to be finite [31]. Moreover, the underlying flow pattern of the air, the viscosity of the lubricant, and the surface topography should lead to nonconstant values of the local slip length [32]. For the physical understanding of slippage at superhydrophobic surfaces that would allow designing optimal surface properties, it is essential to explore the microscopic details of the local flow.

In this Letter, we present an extensive study of the underlying mechanisms of slippage at topographically structured, air-entrapping surfaces. We show that the local slip length, as defined by a Navier slip condition, has a nonconstant, anisotropic distribution following from the flow pattern of the lubricating fluid and furthermore depends on surface-active substances adhering to the water-air interface. We obtain further insight into the physics of slippage by developing a numerical model that considers the local flow field of the entrapped air. Additionally, we discuss the implications of our new findings on the currently accepted models.

As a model surface, we used a square pattern of pillars of $11.5 \mu \mathrm{m}$ in diameter and a spacing of $20 \mu \mathrm{m}$ (Fig. 1), prepared by photolithography of SU8 photoresist on a glass microscope slide and subsequent coating with $1.1 \mu \mathrm{m}$ sized polystyrene beads and fluorinazation with $1 \mathrm{H}, 1 \mathrm{H}, 2 \mathrm{H}, 2 \mathrm{H}-$ perfluorooctyltrichlorosilane [33]. The surface is superhydrophobic (static contact angle $160^{\circ}$ ), which is, however, not strictly needed for slippage. The essential feature is that water placed on the surface stays in the Cassie state and encloses air in between the pillars. By creating overhangs [34], the beads on the top faces ensure a stable Cassie state even under the stress exerted by the flow [35].

The structured surface constitutes the upper wall of a microchannel of $150 \mu \mathrm{m}$ height, $4 \mathrm{~mm}$ width, and $50 \mathrm{~mm}$ length [Fig. 1(c); for details on the microchannel, see [36,37] and Supplemental Material [38]]. Fluorescence 


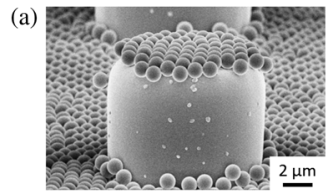

(b)
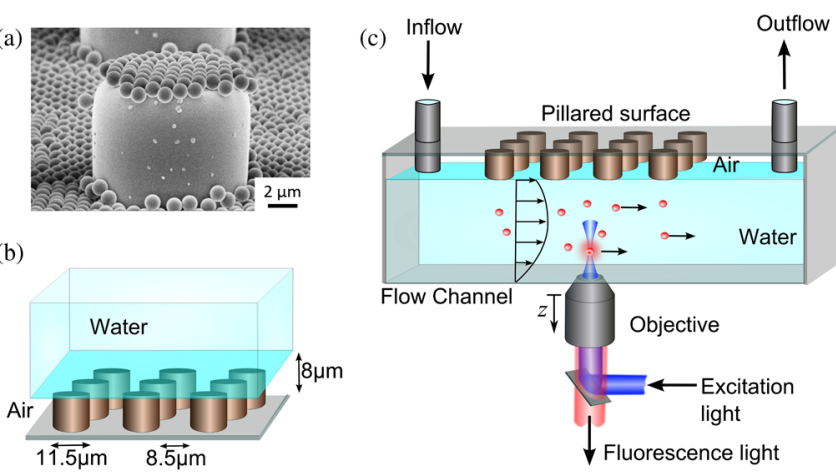

FIG. 1. (a) Scanning electron microscopy image of a pillar of the model surface. (b) Schematic representation of the surface topography. (c) Schematic of the experimental setup.

correlation spectroscopy (FCS) [36,40-45] was used to measure the flow and to deduce the slip length with a high local specificity. As tracers, we used Qdot 585 ITK $^{\text {TM }}$ carboxyl quantum dots (Invitrogen) with a hydrodynamic radius of $R_{h}=6.3 \mathrm{~nm}$. In order to prevent possible electrostatic repulsion of the quantum dots from the fluidic interfaces, a $0.1 \mathrm{mM} \mathrm{K} \mathrm{HPO}_{4}$ solution (Sartorius Arium purified water) was used, corresponding to a Debye shielding length of $\lambda_{D} \approx 17 \mathrm{~nm}$. Before applying this solution, the system was flushed with purified water for $30 \mathrm{~min}$.

By using a hydrostatic pressure difference, the solution was pumped through the microchannel at a flow rate of $35 \mathrm{~mm}^{3} / \mathrm{s}$, corresponding to a Reynolds number of about 9 for the bulk channel flow of the water and about 0.02 for the flow of the air in the lubrication layer. After ensuring a stable Cassie state, flow velocity profiles were collected with a combined FCS-confocal microscopy setup [Carl Zeiss (Germany), Axiovert 200 inverted microscope, LSM510 and ConfoCor 2 modules, C-Apochromat 40×, NA 1.2 water-immersion objective]. At various positions along the air-water interface [Fig. 2(a)], FCS autocorrelation curves were recorded and evaluated to yield the flow velocity at the respective position of the detection volume (for details, see Supplemental Material [38]). The exact position of the pillar-water and the air-water interfaces was determined for each velocity profile by the highest gradient in the measured fluorescence intensity as the detection volume gradually crosses the interface, yielding a vertical accuracy of $\pm 0.3 \mu \mathrm{m}$. Because of the strong surface tension, the water-air interface remains relatively flat under flow, showing a maximum deflection of $<1 \mu \mathrm{m}$. The coordinate $z$ is defined perpendicular to the surface, with $z=0$ referring to the top of the pillars.

Typical profiles for the velocity $u$ are shown in Fig. 3. When the FCS detection volume significantly enters a pillar or air, the tracer concentration and excitation are undefined, and hence measured velocities do not reflect the actual values. Therefore, velocities at positions $<1 \mu \mathrm{m}$ from the interface have been discarded.

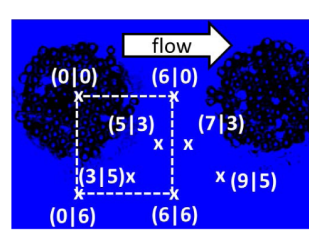

(a)

\begin{tabular}{ll|ll}
\hline Point & $b_{\text {local, exp }}$ & Point & $b_{\text {local, exp }}$ \\
\hline$(0 \mid 0)$ & $0.2 \pm 0.2$ & $(5 \mid 3)$ & $2.4 \pm 0.4$ \\
$(6 \mid 0)$ & $0.3 \pm 0.2$ & $(7 \mid 3)$ & $2.1 \pm 0.3$ \\
$(0 \mid 6)$ & $14.0 \pm 4.9$ & $(3 \mid 5)$ & $7.1 \pm 0.9$ \\
$(6 \mid 6)$ & $4.4 \pm 0.5$ & $(9 \mid 5)$ & $8.5 \pm 1.4$ \\
\hline
\end{tabular}

(b)
FIG. 2. (a) Confocal microscopy image of the air-water interface (blue color corresponds to strong reflection signal) and the coated pillars. Measurement positions are indicated in a pillarreferred coordinate system. (b) FCS-measured $b_{\text {localexp }}(\mu \mathrm{m})$. Uncertainties refer to a given interface height.

The local slip length, which, e.g., is used in theoretical models, is defined by $b_{\text {local,th }}=\left.[u /(\partial u / \partial z)]\right|_{\text {surface }}$. Because of the general experimental challenge to measure velocities in proximity to an interface, this quantity can be assessed only from the velocity profile at a certain distance from the interface. Here, fitting a linear function to the end of the velocity profile (red line in Fig. 3) and extrapolating to $u=0$ below the local position of the interface leads to what we call the experimentally assessable local slip length $b_{\text {local,exp }}$, whose relation to the theoretical one will be explored in the course of this work. Repeated measurements of $b_{\text {local,exp }}$ at the various positions showed a good repeatability with the maximum standard deviation occurring at point $(0 \mid 6)\left(\sigma_{b, \max }=0.7 \mu \mathrm{m}\right)$. In agreement with slip measurements on flat hydrophobic surfaces [2-9], $b_{\text {local,exp }}$ almost vanishes at the pillar-water interface as within the accuracy of the measurements and the roughness due to the polystyrene particles.

To further explore the local flow phenomena that determine the local slip, flow over a similar surface was calculated numerically. The model considers a 3D, periodic surface element. For simplification, the top of the pillar as well as the air-water interface was modeled to be flat, which is in reasonable accordance with the experimental observations. The net flux through the air layer is zero,

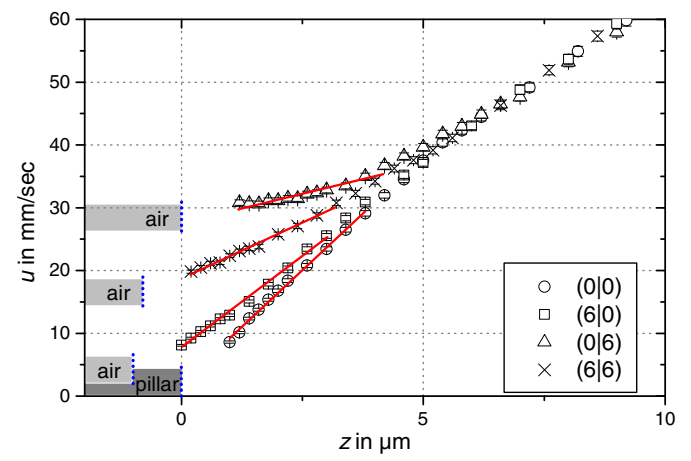

FIG. 3. Exemplary velocity profiles with a fit for the determination of $b_{\text {local,exp }}$ at points $(0 \mid 0),(6 \mid 0),(0 \mid 6)$, and (6|6). Dotted lines indicate the individual position of the interface as detected from the fluorescence intensity profiles. 
accounting for the fact that, in the experiment, the air layer is not connected to the environment but enclosed in the channel. The Stokes equations were solved with the commercial finite-element solver Comsol Multiphysics (for details, see Supplemental Material [38]).

For the flow of the water, the computations yield velocity profiles [Fig. 4(a)] that correspond well to the measured profiles. In addition, calculations also reveal the flow within the air layer. Since the air is enclosed in the channel and has to fulfill mass conservation, it always has a backward-facing component and recirculates in a vortex behind the pillars. Also note the complexity of the flow field within the first micrometers above the interface, where the different interface conditions lead to different curvatures of the velocity profiles. When the profiles even out at a certain distance from the interface, the flow over the pillar, where the no-slip boundary condition is valid, may be ahead of the flow over the interface, which is due to mass conservation in the water.

Both the experiments and calculations show that $b_{\text {local }}$ varies significantly along the interface [Figs. 2(b) and 4(b)].

In fact, we can understand $b_{\text {local }}$ as a model parameter that is defined by the local flow field through the Navier slip condition. Its properties and distribution are consequently determined by the flow within the air layer and close to the surface, which obeys the local hydrodynamics with continuity of mass and stress at the interface. As shown, this flow field is strongly position dependent. Correspondingly, the local slip length is highest where the air is allowed to flow freely between the pillars and low behind the pillar. Since the shear stress varies only slightly, $b_{\text {local }}$ mainly scales with $u$. The computations illustrate that, due to mass conservation in the air layer, the velocity at the interface is highest at the narrowest confinement between the pillars $(0 \mid 6)$, which corresponds to the point of largest local slip. The experimentally measured local slip

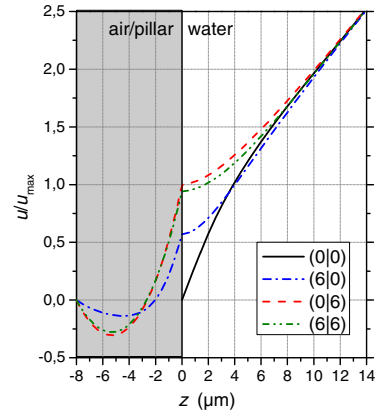

(a)

\begin{tabular}{lll} 
Point & $\begin{array}{l}b_{\text {local, th }} \\
(\mu \mathrm{m})\end{array}$ & $\begin{array}{l}b_{\text {cp. }} \\
(\mu \mathrm{m})\end{array}$ \\
\hline$(0 \mid 0)$ & 0 & 0 \\
$(6 \mid 0)$ & 56 & 4 \\
$(5 \mid 3) /(7 \mid 3)$ & 77 & 7 \\
$(3 \mid 5) /(9 \mid 5)$ & 89 & 12 \\
$(0 \mid 6)$ & 91 & 15 \\
$(6 \mid 6)$ & 99 & 15 \\
\hline
\end{tabular}

(b)
FIG. 4. Numerically calculated flow phenomena close to the surface. The interface deflection is not considered. (a) Velocity profiles at selected positions; the velocity is normalized with the maximum velocity at the interface $u_{\max }$; (b) local slip length in the direction of flow $b_{\text {local,th }}(z=0)$ and local slip length defined for comparison (index cp) with experiments $b_{\mathrm{cp}}$ (observed slip length at $z=c g$ ). distribution is reasonably symmetric $[(5 \mid 3)$ vs $(7 \mid 3)$ and (3|5) vs $(9 \mid 5)]$, confirming that the flow is in the lowReynolds-number regime.

Furthermore, both the experimental and numerical results reveal an important property of the local slip length: It is anisotropic. Comparison of points (0|6) and (6|0), and $(3 \mid 5)$ and $(5 \mid 3)$, respectively, shows that the local slip is a function of the flow direction. The anisotropy is directly linked to the different flow patterns at the individual locations, e.g., strongly recirculating at $(6 \mid 0)$ while flowing less inhibited at $(0 \mid 6)$. While it is well known that the effective slip length may be anisotropic [46,47], e.g., for grooved surfaces, the local slip length has typically been considered to be isotropic [19-30].

For a quantitative comparison of the experimental and numerical local slip values, it is important to note that according to the numerical calculations, above the pillar, the velocity profile in the water is approximately linear close to the interface, whereas above the air-water interface, the velocity profile is nonlinear. Therefore, the experimentally assessable local slip length $b_{\text {local,exp }}$, which was determined from the flow profile at a certain distance from the interface, is not identical to $b_{\text {local,th }}$. For a proper comparison, we can, however, define from the calculations $b_{\mathrm{cp}}=\left.[u /(\partial u / \partial z)]\right|_{z=d}-d$, which corresponds to the local slip length that is observed at the center of gravity $d=2.3 \mu \mathrm{m}$ of the fit range of the experimental data (see Supplemental Material [38]) under the assumption of a linear, experimental-like fit.

In the region where the flow is not obstructed by the pillars (0|6), $b_{\text {local,exp }}$ and $b_{\text {cp }}$ agree well [Figs. 2(b) and 4(b)]. The local slip phenomena are accurately represented by the pure hydrodynamics of the two fluids. In contrast, behind the pillars, the velocity and local slip length are much smaller in the experiments than in the theoretical prediction. This indicates that further processes influence the slip on the microscopic level, for example, interfacial impurities [31]. In a system with such a high surface-tovolume ratio as studied here, a complete absence of any surface impurity cannot be guaranteed, although we worked as clean as possible.

In order to clarify the role of fluid-fluid interface impurities, the sensitivity of the local slip length to the addition of a surfactant is investigated. As a surfactant, sodium 1-decanesulfonate (S-1DeS) (Sigma Aldrich) is employed, which at low concentrations allows for comparatively small changes in the surface tension $[66 \mathrm{mN} / \mathrm{m}$ at $0.1 \mathrm{cmc}$ (critical micelle concentration $47 \mathrm{mmol} / \mathrm{l}$ ) and $50 \mathrm{mN} / \mathrm{m}$ at $0.4 \mathrm{cmc}$ in water, Dataphysics DCAT11EC tensiometer]. Hence, the water-air interface remains stable and close to flat as confirmed by confocal imaging.

The local slip length is clearly decreased by the presence of surfactants [Fig. 5(a)]. Because of the nature of the flow field, surfactants will first accumulate in front of the pillars and drive a backflow due to Marangoni forces, which is in 


\begin{tabular}{lrr} 
(a) & & \\
\hline S-1DeS & $0.1 \mathrm{cmc}$ & $0.4 \mathrm{cmc}$ \\
\hline$(0 \mid 0)$ & $0.1 \pm 0.2$ & $-0.1 \pm 0.2$ \\
$(6 \mid 0)$ & $0.2 \pm 0.2$ & $0.4 \pm 0.2$ \\
$(0 \mid 6)$ & $5.3 \pm 1.0$ & $4.6 \pm 0.9$ \\
$(6 \mid 6)$ & $3.9 \pm 0.5$ & $3.2 \pm 0.8$ \\
\hline
\end{tabular}

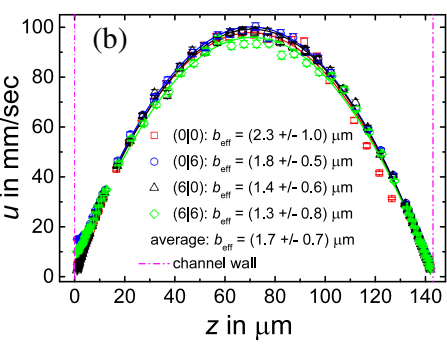

FIG. 5. (a) $b_{\text {local, exp }}(\mu \mathrm{m})$ depending on the surfactant concentration. (b) FCS-measured velocity profiles across the channel with a parabolic fit.

line with our observation that there is hardly any flow at (6|0). Consequently, concentration-induced flows due to surface active substances lead to an effective no-slip boundary condition. We expect that even small amounts of contaminations of the water-air interface can immobilize the motion at the interface and thereby negatively affect slippage at superhydrophobic surfaces. Also, slippery surfaces that are based on an alternative lubricating fluid, like oil, could be affected. A similar behavior based on surfactant-concentration gradients is known to occur for bubbles [48]. Being effectively always present, surface active substances may be crucial for the applicability of superhydrophobic surfaces.

Furthermore, we investigated the effective slip length $b_{\text {eff }}$. While flow close to a structured wall varies according to the local boundary conditions, these fluctuations die off far from the wall, and the flow attains an average velocity profile. For the present channel geometry, where the pillar period is much smaller than the channel height and the channel height than its width, the average velocity profile is parabolic with a no-slip condition at the unstructured, hydrophilic, lower wall and an effective slip at the superhydrophobic upper wall. The effective slip length was determined by recording velocity profiles across the entire channel and fitting them to a theoretical parabolic profile. A region of the order of the pillar period was excluded from the fit, making sure that the changes due to the varying wall structure have died off. For consistency, measurements of the effective slip length are performed at various positions [Fig. 5(b)]. On average, we measured an effective slip length of $b_{\text {eff }}=1.7 \pm 0.7 \mu \mathrm{m}$.

In the numerical model, the effective slip length $b_{\text {eff,th }}$ was obtained by matching the computed flow far from the structured wall with the theoretical profile for a flow with an effective slip length at the wall. With $b_{\text {eff,th }}=4 \mu \mathrm{m}$, the theoretical value is about 2.5 times larger than the measured one. Since the theoretical model does not consider any influence of interface contaminations, the numerical value should be larger than the experimental one. Still, the experimental and numerical values for the effective slip length are closer to each other than the corresponding comparison of the local values would suggest [Figs. 2(b) and 4(b)]. This stems from the fact that the characteristic local slip length of a classic superhydrophobic surface is quite large. The larger the local slip length, the less sensitive the effective slip length is to the exact local processes [32]. For the present surface, assuming an infinite local slip length at the airwater interface theoretically leads to an effective slip length of $4.3 \mu \mathrm{m}$.

In this Letter, we investigated the hydrodynamics of slippage at a microstructured surface in the Cassie state both experimentally by FCS measurements as well as theoretically by numerical calculations. We showed that the local flow pattern close to the interface is complex and strongly varying as a function of the surface topography and surfactants adhering to the air-water interface, which is crucial for experiments to take into account. The local hydrodynamics determine the effective and local slippage as commonly used in theoretical models. We found the latter parameter has a finite, position-dependent, and anisotropic distribution. These properties of the local flow field and slip length provide a path to appropriate modeling and, hence, to the physical understanding of superhydrophobic and related surfaces. They are also of interest for drop motion and directional splashing on superhydrophobic surfaces $[49,50]$. Since current theoretical models use an isotropic, infinite or constant finite local slip length, it has to be evaluated to what extent such simple assumptions may reasonably be employed for the description of superhydrophobic or slippery surfaces. As we discussed, the effective slip length of surfaces exhibiting a large local slip length is not very sensitive to changes in the local properties. With respect to the purely global, effective behavior of the flow, traditional models based on an infinite or isotropic local slip may consequently lead to similar results for the effective slip length or the overall drag despite the fact that the local processes are not well captured. If surface active substances regionally or generally block the motion in the lubricating layer and thereby significantly reduce the local slip length, new models are required that take into account the properties of the local slip length. The same applies to surfaces with a small local slip length, e.g., due to a high viscosity of the lubricating medium, or when the geometry of the surface has a significant influence on the flow field close to the air-water interface. Apart from the global flow behavior, the correct modeling of local flow behavior relies on correct assumptions for the local slip length. This may, for example, be important for turbulent flow over slippery surfaces. With increasing Reynolds numbers, the vortices forming in the air should become asymmetric, leading to a different flow field and a different slip length distribution. To properly describe such cases, further investigations are needed.

The authors thank Lena Mammen and Noemí Encinas García for the preparation of the pillars and Franziska Henrich for the surface tension measurements of S-1DeS. 
We also thank the Polymer Theory Group at MPIP Mainz for access to the cluster computing facilities. Financial support from ERC (Advanced Grant No. 340391-SUPRO) and HFSP (RGP0013/2015) is gratefully acknowledged.

*schoenecker@mpip-mainz.mpg.de

[1] M. Navier, Mem. Acad. R. Sci. Inst. France 6, 389 (1823).

[2] R. Pit, H. Hervet, and L. Léger, Tribol. Lett. 7, 147 (1999).

[3] J. Baudry, E. Charlaix, A. Tonck, and D. Mazuyer, Langmuir 17, 5232 (2001).

[4] V. S. J. Craig, C. Neto, and D. R. M. Williams, Phys. Rev. Lett. 87, 054504 (2001).

[5] E. Bonaccurso, M. Kappl, and H.-J. Butt, Phys. Rev. Lett. 88, 076103 (2002).

[6] C. Cottin-Bizonne, B. Cross, A. Steinberger, and E. Charlaix, Phys. Rev. Lett. 94, 056102 (2005).

[7] P. Joseph and P. Tabeling, Phys. Rev. E 71, 035303 (2005).

[8] D. Lasne, A. Maali, Y. Amarouchene, L. Cognet, B. Lounis, and H. Kellay, Phys. Rev. Lett. 100, 214502 (2008).

[9] E. Lauga, M. P. Brenner, and H. A. Stone, in Handbook of Experimental Fluid Dynamics, edited by J. Foss, C. Tropea, and A. Yarin (Springer, New York, 2005).

[10] E. Schnell, J. Appl. Phys. 27, 1149 (1956).

[11] K. Watanabe, Y. Udagawa, and H. Udagawa, J. Fluid Mech. 381, 225 (1999).

[12] P. Tsai, A. M. Peters, C. Pirat, M. Wessling, R. G. H. Lammertink, and D. Lohse, Phys. Fluids 21, 112002 (2009).

[13] G. McHale, N. J. Shirtcliffe, C. R. Evans, and M. I. Newton, Appl. Phys. Lett. 94, 064104 (2009).

[14] N. J. Shirtcliffe, G. McHale, M. I. Newton, and Y. Zhang, ACS Appl. Mater. Interfaces 1, 1316 (2009).

[15] H. Park, G. Sun, and C.-J. C. Kim, J. Fluid Mech. 747, 722 (2014).

[16] B. R. Solomon, K. S. Khalil, and K. K. Varanasi, Langmuir 30, 10970 (2014).

[17] D. Song, R. Daniello, and J. Rothstein, Exp. Fluids 55, 1783 (2014).

[18] S. Srinivasan, J. A. Kleingartner, J. B. Gilbert, R. E. Cohen, A. J. B. Milne, and G. H. McKinley, Phys. Rev. Lett. 114, 014501 (2015).

[19] E. Lauga and H. A. Stone, J. Fluid Mech. 489, 55 (2003).

[20] J. Davies, D. Maynes, B. W. Webb, and B. Woolford, Phys. Fluids 18, 087110 (2006).

[21] M. Sbragaglia and A. Prosperetti, Phys. Fluids 19, 043603 (2007).

[22] A. M. J. Davis and E. Lauga, Phys. Fluids 21, 011701 (2009).

[23] D. Crowdy, Phys. Fluids 22, 121703 (2010).

[24] A. M. J. Davis and E. Lauga, J. Fluid Mech. 661, 402 (2010).

[25] S. Türk, G. Daschiel, A. Stroh, Y. Hasegawa, and B. Frohnapfel, J. Fluid Mech. 747, 186 (2014).

[26] A. V. Belyaev and O. I. Vinogradova, J. Fluid Mech. 652, 489 (2010).
[27] A. V. Belyaev and O. I. Vinogradova, Phys. Rev. Lett. 107, 098301 (2011).

[28] K. Kamrin, M. Z. Bazant, and H. A. Stone, J. Fluid Mech. 658, 409 (2010).

[29] E. S. Asmolov and O. I. Vinogradova, J. Fluid Mech. 706, 108 (2012).

[30] E. S. Asmolov, S. Schmieschek, J. Harting, and O. I. Vinogradova, Phys. Rev. E 87, 023005 (2013).

[31] G. Bolognesi, C. Cottin-Bizonne, and C. Pirat, Phys. Fluids 26, 082004 (2014).

[32] C. Schönecker, T. Baier, and S. Hardt, J. Fluid Mech. 740, 168 (2014).

[33] L. Mammen, K. Bley, P. Papadopoulos, F. Schellenberger, N. Encinas, H.-J. Butt, C. K. Weiss, and D. Vollmer, Soft Matter 11, 506 (2015).

[34] S. Herminghaus, Europhys. Lett. 52, 165 (2000).

[35] J. S. Wexler, I. Jacobi, and H. A. Stone, Phys. Rev. Lett. 114, 168301 (2015).

[36] O. I. Vinogradova, K. Koynov, A. Best, and F. Feuillebois, Phys. Rev. Lett. 102, 118302 (2009).

[37] S. Yordanov, A. Best, H.-J. Butt, and K. Koynov, Opt. Express 17, 21149 (2009).

[38] See Supplemental Material at http://link.aps.org/ supplemental/10.1103/PhysRevLett.116.134501 for details on the microfluidic channel, the measurement procedure and the numerical model, which includes Refs. [22,37, 39-41].

[39] D. Magde, W. W. Webb, and E. L. Elson, Biopolymers 17, 361 (1978).

[40] M. Gösch, H. Blom, J. Holm, T. Heino, and R. Rigler, Anal. Chem. 72, 3260 (2000).

[41] Fluorescence Correlation Spectroscopy, edited by R. Rigler and E. Elson (Springer, New York, 2001).

[42] D. Magde, E. Elson, and W. W. Webb, Phys. Rev. Lett. 29, 705 (1972).

[43] D. Lumma, A. Best, A. Gansen, F. Feuillebois, J. O. Rädler, and O. I. Vinogradova, Phys. Rev. E 67, 056313 (2003).

[44] K. Koynov and H.-J. Butt, Curr. Opin. Colloid Interface Sci. 17, 377 (2012).

[45] D. Schaeffel, S. Yordanov, M. Schmelzeisen, T. Yamamoto, M. Kappl, R. Schmitz, B. Dünweg, H.-J. Butt, and K. Koynov, Phys. Rev. E 87, 051001 (2013).

[46] H. Stone, A. Stroock, and A. Ajdari, Annu. Rev. Fluid Mech. 36, 381 (2004).

[47] M. Z. Bazant and O. I. Vinogradova, J. Fluid Mech. 613, 125 (2008).

[48] V. G. Levich, Physicochemical Hydrodynamics (PrenticeHall, Englewood Cliffs, NJ, 1962).

[49] M. Reyssat, D. Richard, C. Clanet, and D. Quere, Faraday Discuss. 146, 19 (2010).

[50] P. Tsai, R. C. A. van der Veen, M. van de Raa, and D. Lohse, Langmuir 26, 16090 (2010). 